\title{
Endovascular Thrombolysis for Severe Cerebral Sinus Thrombosis
}

\author{
Y. PENG, J-G. XUAN, Y-L. YANG, S-N. WANG \\ The first Hospital of Changzhou City, Suzhou University, Changzhou City, China
}

Key words: cerebral sinus thrombosis, thrombolysis

\section{Summary}

Cerebral venous and sinus thrombosis (CVST) is a rare but life-threatening disease accounting for less than 1\% of all strokes and a poor outcome in around 10\% of all patients. Early diagnosis is crucial because anticoagulation may reduce the risk of a fatal outcome and severe disability. However, the absence of specific features and the possibility of presentation without associated neurological signs can make its early recognition difficult. For these CVST patients with deteriorating symptoms, endovascular thrombolysis might give better outcome. We describe our five cases of CVST with clinical deteriorating symptoms treated with endovascular thrombolysis. Four patients made an excellent recovery, whereas one patient died despite maximal treatment including decompressive craniectomy.

\section{Introduction}

Cerebral venous and sinus thrombosis (CVST) is an uncommon but potentially lethal event. The mortality rate of CVST is still $10 \%$ and surviving patients may suffer significant morbidity such as seizures or neurological deficits ${ }^{1,2}$. Early diagnosis is crucial because anticoagulation may reduce the risk of a fatal outcome and severe disability. However, the absence of specific features and neurological signs or a negative brain CT can make its early recognition difficult and physicians are always less likely to consider the possibility of CVST in patients with recent headache. When deteri- orating symptoms or neurologic deficits present, a standard treatment with heparin and anticoagulation is insufficient for a good outcome. For these patients, endovascular thrombolytic therapy is a more effective method.

In this retrospective series, we describe our experience of five severe sinus thrombosis cases treated with endovascular thrombolysis.

\section{Materials and Methods}

From May 2006 to June 2008, five patients with cerebral sinus thrombosis were treated by endovascular thrombolysis at The First Hospital of Changzhou City, Suzhou University. All patients were transferred from other hospitals. Among them, three were females including one pregnancy of 16 weeks. The mean age of patients was 27 years (range 17 to 41). Clinical symptoms included aggravating headache, nausea and vomiting in all cases, seizures in one case, neurological deficits in two and coma in two. The mean days between onset to diagnosis were 14.2 days. Computed tomography (CT) was performed in all cases. Magnetic resonance imaging (MRI) and time of flight magnetic resonance venography (MRV) were performed in three patients.

All cases received digital subtraction angiography. These data demonstrated superior sagittal sinus (SSS) thrombosis in four cases and straight sinus thrombosis in one. The scans of pretreatment CT and MRI revealed hemorrhagic infarct in the bilateral parietal region in case 3. Ancillary laboratory workup revealed unremarkable findings in all cases. The in- 
tracranial pressure (ICP) pretreatment was 520 $\mathrm{mm} \mathrm{H}_{2} \mathrm{O}$ and $460 \mathrm{~mm} \mathrm{H}_{2} \mathrm{O}$ respectively in case 1 and case 2 .

Three patients received therapeutic anticoagulation with unfractionated intravenous heparin for two days and received thrombolytic therapy due to clinical deterioration. Two cases were treated with thrombolysis in emergency because of coma. We applied thrombolysis by introducing a microcatheter via the internal jugular vein and advancing it into the superior sagittal sinus or the straight sinus while the thrombus was dissolved with urokinase (bolus 500 to $800 \times 103$ IU).

The microcatheter was left in the sinus and continuing urokinase was infused locally at a rate of $800 \times 103 \mathrm{IU} / \mathrm{d}$ for one to four days. Two cases were combined with balloon angioplasty with balloon catheter (HyperGlide, ev3, Inc.). Systemic heparinization was given during the procedure until the microcatheter was taken out. Recovery was continued with warfarin for three to six months with a target international normalized ratio of 2.0-2.5.

\section{Results}

Four patients recovered well with no residual symptoms when discharged. The intracranial pressure (ICP) decreased to $180 \mathrm{~mm} \mathrm{H}_{2} \mathrm{O}$ and $150 \mathrm{~mm} \mathrm{H}_{2} \mathrm{O}$ in case 1 and case 2 at day seven post-thrombolysis. One patient died from transtentorial herniation although we performed decompressive craniectomy. The Glasgow Outcome Scale of all cases are showed in Table 1. Six months follow-up time of flight MRV showed complete recanalization of the sinus in three cases. All data of patients are summarized in Table 1.

\section{Illustrative Cases}

Case 1. A 17-year-old male high school student had aggravating headache, nausea and vomiting for 12 days and was examined in a local hospital. He was brought to our hospital due to aggravating headache and deteriorating vision. CT and MRI showed higher density areas in the posterior SSS (Figure 1A) and time of flight MRV showed an occluded posterior SSS (Figure 1B). The intracranial pressure (ICP) was $520 \mathrm{~mm} \mathrm{H}_{2} \mathrm{O}$. Despite therapeutic anticoagulation with unfractionated intravenous heparin for two days, his vision continued to deteriorate. We decided to treat him with local thrombolysis by a bolus of urokinase $800 \times 103$ IU directly through the clot via microcatheter (Figure 1D). The microcatheter was left in the sinus and continuing urokinase was infused locally at a rate of $800 \times 103 \mathrm{IU} / \mathrm{d}$ for three days. Angiography preprocedure and post-procedure revealed occlusion of the posterior third of the SSS (Figure $1 \mathrm{C}$ ) and most partial recurrence (Figure 1E). His headache gradually disappeared and the ICP reduced to $180 \mathrm{~mm} \mathrm{H}_{2} \mathrm{O}$ on day seven postthrombolysis. He was discharged with warfarin for six months with a target international normalized ratio of 2.0-2.5. He had no deficit at one month follow-up. Time of flight MRV at six months showed complete recanalization of the sinus (Figure 1F).

Figure 1 A) Sagittal T-1 weighted MR shows high signal thrombus within posterior SSS. B) Sagittal slab of MRV shows signal drop out within the occluded posterior SSS. C) Lateral angiogram shows absent flow in the posterior SSS. D) Anteroposterior angiogram shows microcatheter in the thrombus. E) Lateral angiogram immediately after initial bolus $800 \times 103$ IU urokinase shows most partially recanalization in the posterior SSS. F) Time of flight MRV at six months follow-up shows complete recanalization of the SSS.

Table 1 Clinic Data of 5 patients With delayed diagnosed CST.

\begin{tabular}{|c|c|c|c|c|c|c|c|c|}
\hline No. & $\begin{array}{l}\text { Patient } \\
\text { Sex }\end{array}$ & Age & $\begin{array}{c}\text { Risk } \\
\text { Factor }\end{array}$ & $\begin{array}{l}\text { Onset to } \\
\text { Diagnosis } \\
\text { (days) }\end{array}$ & $\begin{array}{c}\text { Diagonosis to } \\
\text { Thrombolysis } \\
\text { (days) }\end{array}$ & $\begin{array}{c}\text { GCS } \\
\text { Pretreatment }\end{array}$ & $\begin{array}{c}\text { Neurologic } \\
\text { Deficits }\end{array}$ & $\begin{array}{l}\text { Outcome } \\
\text { GOS }\end{array}$ \\
\hline 1 & M & 17 & -- & 12 & 2 & 15 & deteriorating vision & I \\
\hline 2 & M & 17 & head injure & 10 & 2 & 15 & mental alertness & I \\
\hline 3 & $\mathrm{~F}$ & 24 & pregnancy & 15 & 0 & 6 & coma, right hemiparesis & V \\
\hline 4 & $\mathrm{~F}$ & 35 & -- & 10 & 0 & 8 & coma & I \\
\hline 5 & $\mathrm{~F}$ & 41 & -- & 24 & 2 & 10 & seizure & I \\
\hline
\end{tabular}




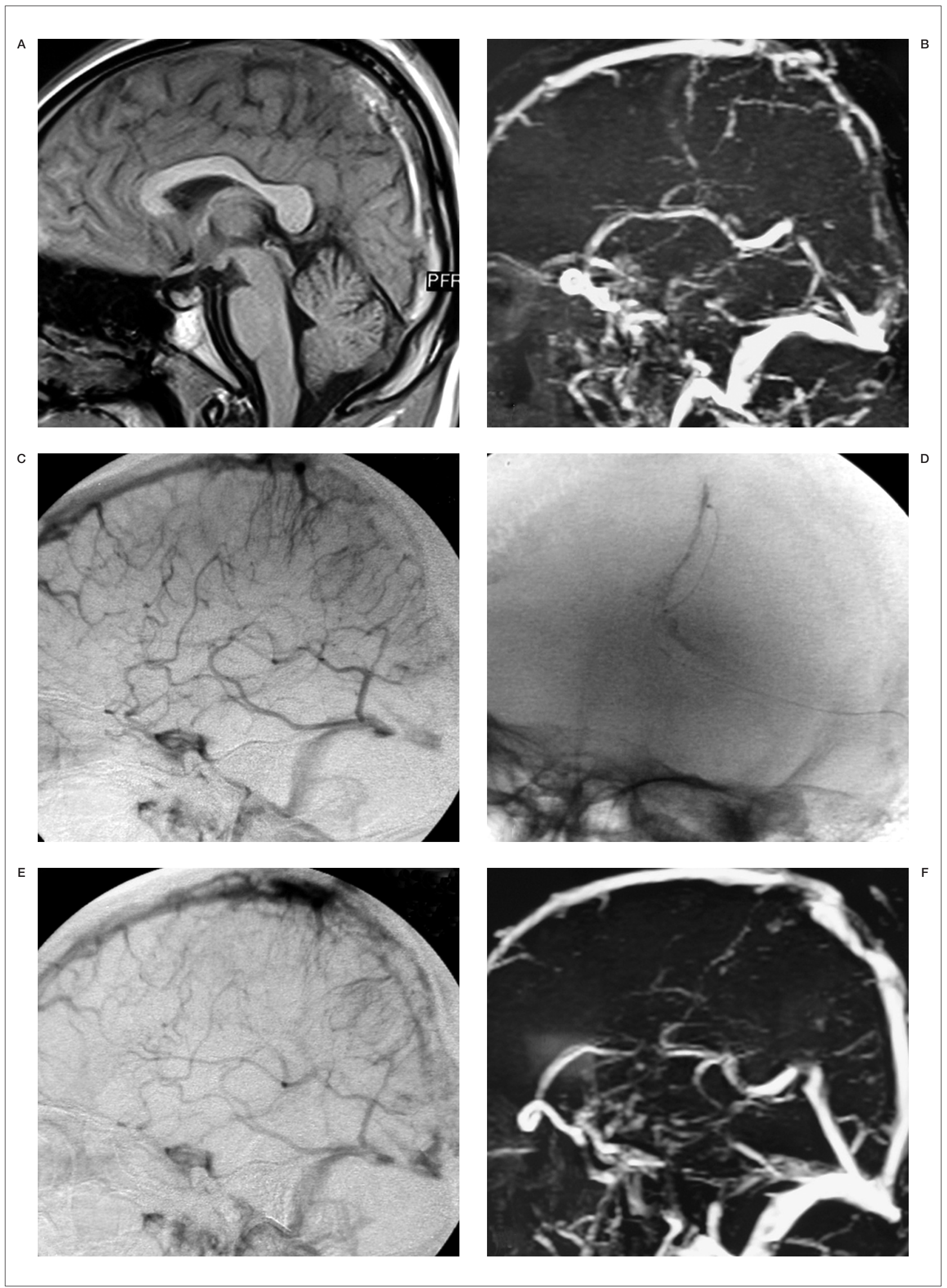




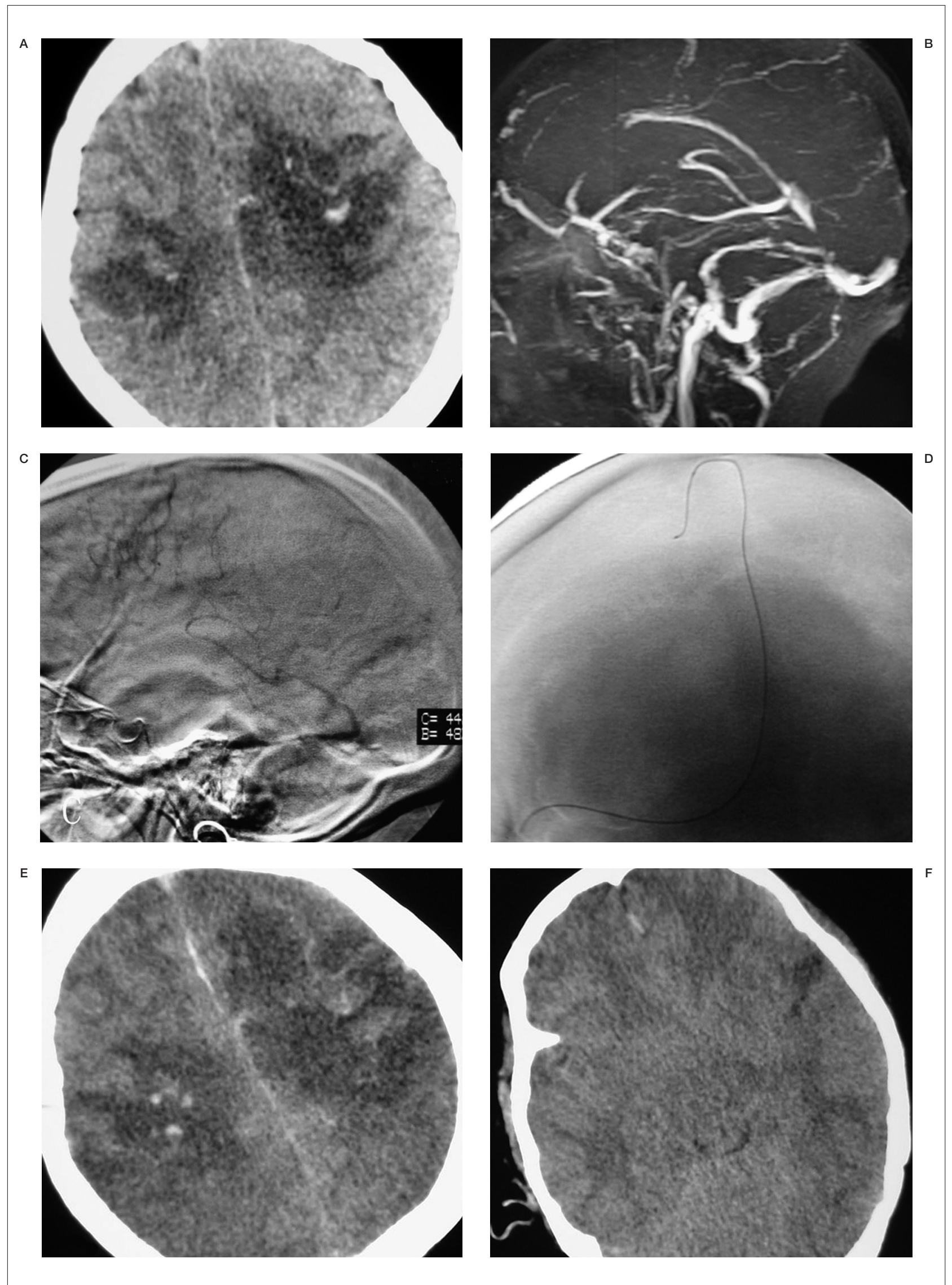


Case 2. A 24-year-old woman who was 16 weeks pregnant was referred to our emergency room due to unconsciousness. Her husband and parents complained she had headache and vomiting for two weeks month but had ignored it as the reaction of pregnancy. She was comatose and had right hemiparesis. GCS score was 6. CT showed hemorrhagic infarct in bilateral parietal region (Figure 2A) and time of flight MRV showed a whole occluded SSS (Figure 2B). Acute thrombolytic therapy with urokinase and angioplasty were started immediately (D). We performed $800 \times 103$ IU urokinase but still thrombus persisted. A $4 \times 15 \mathrm{~mm}$ compliant occlusion microballoon catheter (HyperGlide, ev3, Inc.) was inserted into the SSS, expanded, and then retracted through the SSS. Multiple passes were performed. Contrast injection after the balloon angioplasty procedure demonstrated significant flow improvement. Angiography demonstrated the occlusion of the whole SSS (Figure 2C) and post-procedure angiography showed partial recurrence (picture not shown). The microcatheter was left in the sinus and continuing urokinase was infused at a rate of $800 \times 103 \mathrm{IU} / \mathrm{d}$. Her left pupil fixed and dilated at one day post-thrombolysis. No rebleeding, enlarged infarction, edema or obliterated basal cisterns were found on CT scans (Figure E,F). We performed decompressive craniectomy. A large left frontotemporoparietal flap was created, a wide bone flap was removed, the dura was opened and an autologous pericranial tissue graft was sutured loosely in place for dural augmentation. However, the patient died four days after admission.

\section{Discussion}

Cerebral venous and sinus thrombosis (CVST) has no pathognomonic signs or symptoms. The most common signs and symptoms are headache, seizures, focal neurological deficits, papilledema and altered level of con-

Figure 2 A) CT scan shows hemorrhagic infarct in the bilateral parietal region. B) Sagittal slab of MRV shows signal drop out within the occluded SSS. C) Lateral angiogram shows absent flow in the SSS. D) Anteroposterior angiogram shows location of the microcatheter in the SSS. $\mathrm{E}, \mathrm{F}) \mathrm{CT}$ scans at 1 day post-thrombolysis show enlarged infarction, edema and obliterated basal cisterns. sciousness. Headache is the most frequent and often the earliest symptom of CVST but the absence of specific features makes its recognition difficult ${ }^{3-5}$. In clinical practice diagnosis is still frequently overlooked or delayed due to the wide spectrum of clinical symptoms. However, early diagnosis is crucial since anticoagulation may reduce the risk of a fatal outcome and severe disability ${ }^{6-8}$. All our cases were diagnosed after a mean delay of 14.2 days. They already had one or more risk factors ${ }^{9}$ associated with a poor outcome when they were transferred to our institute.

The diagnosis of CVST is based on neuroimaging. Angiography has long been the gold standard for diagnosis of CVST ${ }^{8}$ which depends on non-visualization of a sinus or vein and other indirect signs such as dilated collateral veins with corkscrew appearance, delayed venous phase, collateral circulation. As a non-invasive method, magnetic resonance imaging and MR venography are regarded the best tools both for the diagnosis and follow-up of CVST ${ }^{10,11}$.

The confirmation of CVST relies on the demonstration of the venous thrombus on MR imaging and lack of patency on MR venography. Computed tomography (CT) is usually the first investigation performed in the emergency department. However, it is normal in $25-30 \%$ of patients and its main value is to rule out other conditions such as stroke and tumors and to reveal signs suggestive of $\mathrm{CVST}^{8,12}$.

In our series, four patients made an excellent recovery, whereas one patient died despite maximal treatment including thrombolysis and decompressive craniectomy. The most conspicuous factors in the patient who died were edema and large bilateral hemorrhagic infarcts causing herniation. This agrees with the study of Canhao et $\mathrm{Al}^{13}$ which demonstrated that causes of early death in CVST are transtentorial herniation due to a mass lesion, diffuse cerebral edema, or multifocal parenchymal hemorrhage.

There was no case of increased hemorrhage after thrombolysis in our study even in the case of bilateral hemorrhagic infarcts pretreatment. We think thrombolytic therapy is safe and effective. Although there is no current evidence from randomized controlled trials on the efficacy and safety of local thrombolytic therapy in patients with CVST, local thrombolytic therapy has been widely used for faster restitution of venous outflow and more positive effects ${ }^{14-17}$. Patients were either treated with heparin and 
urokinase or heparin and recombinant tissue plasminogen activator (rtPA) which may carry fewer bleeding complications due to its clot selectiveness and shorter half-life. As the mean days between onset to diagnosis were 14.2 days in this group, we prefer indwelling microcatheter in the sinus to continue infusing urokinase for one to four days after the first bolus of urokinase. We think this is better for thrombolysis of chronic thrombus ${ }^{18}$. But it is a pity we did not perform further angiography to obtain more information when withdrawing the microcatheter.

The success of mechanical thrombectomy in conjunction with local thrombolysis has been shown to the improve rate of recanalization and reduce the dose of overall thrombolysis ${ }^{19,20}$. New devices such as the Merci device was considered for the treatment of CVST thrombosis more effectively ${ }^{21}$. Since the first case reports of thrombolysis for CVST in $1988^{22}$, all encouraging results of thrombolysis in last 20 years have been based on case reports and uncontrolled case series. In addition, there are still no established clinical criteria for the use of thrombolytics in CVST. A controlled randomized trial is necessary to further study the efficacy and safety of thrombolysis in CVST.

\section{Conclusions}

We present our experience of endovascular thrombolytic treatment of severe cerebral venous and sinus thrombosis with risk factors associated a poor outcome. Although the level of evidence is very limited and based on case reports, local thrombolysis appears to be an alternative life-saving treatment that accelerates recanalization of occluded dural sinus in selected patients with clinical deterioration.

\section{References}

1 Ferro JM, Canhao $\mathrm{P}$ et $\mathrm{Al}$ : Prognosis of cerebral vein and dural sinus thrombosis: results of the International Study on Cerebral Vein and Dural Sinus Thrombosis (ISCVT). Stroke 35: 664-670, 2004.

2 Bousser MG, Ferro JM: Cerebral venous thrombosis: an update. Lancet Neurol 6: 162-170, 2007.

3 Fink JN, McAuley DL: Cerebral venous sinus thrombosis: a diagnostic challenge. Int Med J7: 384-390, 2001.

4 Agostoni E: Headache in cerebral venous thrombosis. Neurol Sci 25 (sup 3): S206- S210, 2004.

5 Stolz E, Rahimi A et Al: Cerebral venous thrombosis: an all or nothing disease? Prognostic factors and longterm outcome. Clin Neurol Neurosurg 107: 99-107, 2005.

6 Stam J, de Bruijn SFTM, deVeber G: Anticoagulation for cerebral sinus thrombosis. Cochrane Database Syst Rev 4: CD002005, 2001.

7 Einhãupl KM, Villringer A et $\mathrm{Al}$ : Heparin treatment in sinus venous thrombosis. Lancet 338: 597-600, 1991.

8 Einhãupl KM, Masuhr F: Cerebral venous and sinus thrombosis-an update. Eur J Neurol 1: 109-126, 1994.

9 Stam J, Majoie CB et Al: Endovascular Thrombectomy and Thrombolysis for Severe Cerebral Sinus Thrombosis A Prospective Study. Stroke 39(5): 1487-1490, 2008.

10 Vogl TJ, Bergmann C et Al: Dural sinus thrombosis: value of venous MR angiography for diagnosis and follow up. Am J Roentgenol 162: 1191-1198, 1994.

11 Conner SEJ, Jarosz JM: Magnetic Resonance Imaging of Cerebral Venous Sinus Thrombosis. Clinic Radiology 57: 449-461, 2002.

12 Bousser MG, Ross Russel R: Cerebral venous thrombosis. vol 1, Saunders, London, 1997.

13 Canhao P, Ferro JM et Al: Causes and predictors of death in cerebral venous thrombosis. Stroke 36: 1720$1725,2005$.

14 Horowitz M, Purdy $\mathrm{P}$ et $\mathrm{Al}$ : Treatment of dural sinus thrombosis using selective catheterisation and urokinase. Ann Neurol 38: 58-67, 1995.
15 Kim SY, Suh JH: Direct endovascular thrombolytic therapy for dural sinus thrombosis: infusion of alteplase. Am J Neuroradiol 18: 639-645, 1997.

16 Frey IL, Muro GJ et Al: Cerebral venous thrombosis: combined intrathrombus rtPA and intravenous heparin. Stroke 30: 489.494, 1999.

17 Wasay M, Bakshi R et Al: Nonrandomized comparison of local urokinase thrombolysis versus systemic heparin anticoagulation for superior sagittal sinus thrombosis. Stroke 32: 2310-2317, 2001.

$18 \mathrm{Li} \mathrm{S}, \mathrm{Li}$ B et Al: Study of treatment methods for cerebral venous thrombosis. Chinese Journal of Minimally Invasive Neurosurgery 11: 293-296, 2006.

19 Opatowsky MJ, Morris PP et Al: Rapid thrombectomy of superior sagittal sinus and transverse sinus thrombosis with a rheolytic catheter device. Am J Neuroradiol 20: 414-417, 1999.

20 Dowd CF, Malek AM et Al: Application of a rheolytic thrombectomy device in the treatment of dural sinus thrombosis: a new technique. Am J Neuroradiol 20: 568-570, 1999.

21 Newman CB, Pakbaz RS et Al: Endovascular treatment of extensive cerebral sinus thrombosis. Case report. J Neurosurg 110: 442-445, 2009.

22 Scott JA, Pascuzzi RM et Al: Treatment of dural sinus thrombosis with local urokinase infusion. Case report. J Neurosurg 68: 284-287, 1988.

Ya Peng, M.D.

The first Hospital

of Changzhou City, Suzhou University

Changzhou City, China

E-mail: wilson-pengya@hotmail.com 